\title{
Moderate Colon Dysplasia
}

National Cancer Institute

\section{Source}

National Cancer Institute. Moderate Colon Dysplasia. NCI Thesaurus. Code C4849.

A morphologic finding indicating the presence of moderate dysplastic cellular changes and moderate architectural changes in the glandular epithelium of the colonic mucosa.

There is no evidence of invasion. 